ESAIM: PROCEEDINGS, August 2009, Vol. 28, p. 80-99

M. Ismail, B. Maury \& J.-F. Gerbeau, Editors

\title{
ROBUST NUMERICAL SCHEMES FOR ANISOTROPIC DIFFUSION PROBLEMS, A FIRST STEP FOR TURBULENCE MODELING IN LAGRANGIAN HYDRODYNAMICS *
}

\author{
Julien Dambrine ${ }^{1}$, Philippe Hoch ${ }^{2}$, RaphaËl $\mathrm{Kuate}^{3}$, Jérôme LohéaC ${ }^{4}$, Jérôme \\ MÉtral $^{2}$, Bernard Rebourcet $^{2}$ and Lisl Weynans ${ }^{1}$
}

\begin{abstract}
Numerous systems of conservation laws are discretized on Lagrangian meshes where cells nodes move with matter. For complex applications, cells shape or aspect ratio often do not insure sufficient accuracy to provide an acceptable numerical solution and use of ALE technics is necessary. Here we are interested with conduction phenomena depending on velocity derivatives coming from the resolution of gas dynamics equations. For that, we propose the study of a mock of second order turbulent mixing model combining an elliptical part and an hyperbolic kernel. The hyperbolic part is approximated by finite-volume centered scheme completed by a remapping step see [7]. A major part of this paper is the discretization of the anisotropic parabolic equation on polygonal distorted mesh. It is based on the scheme described in [9] ensuring the positivity of the numerical solution. We propose an alternative based on the partitioning of polygons in triangles. We show some preliminary results on a weak coupling of hydrodynamics and parabolic equation whose tensor diffusion coefficient depends on Reynolds stresses.

Résumé. De nombreux systèmes de lois de conservation sont intégrés à l'aide du formalisme lagrangien où les sommets des mailles voient leurs position varier au cours du temps. La forme des mailles ne permet pas toujours d'assurer une bonne précision du calcul et les techniques ALE sont nécessaires. Nous nous intéressons ici à des phénomènes de conduction dépendant du gradient de vitesse couplés à la dynamique des gaz. Pour cela, nous proposons l'étude d'un simulacre de modèle de mélange turbulent du second ordre construit pour combiner terme elliptique et noyau hyperbolique. La partie hyperbolique est résolue par des schéma centrés volumes-finis et le remaillage de la phase ALE est celui décrit dans [7]. Une partie importante de ce papier est la discrétisation de l'équation parabolique anisotrope sur maillage polygonal non régulier. Celle-ci s'inspire du schéma dans [9] qui assure la positivité de la solution numérique. Nous proposons une variante basée sur le découpage en triangles des mailles polygonales. Nous montrons quelques résultats préliminaires sur un couplage faible hydrodynamique et équation parabolique dont le tenseur de diffusion dépend des tensions de Reynolds.
\end{abstract}

\footnotetext{
* Special thanks to organizers of Cemracs 08: J.B. Apoung, L. Boudin, M. Ismail, S. Martin, B. Maury, C. Misbah, T. Takahashi

${ }^{1}$ Université Bordeaux 1 351, cours de la Libération F-33405 Talence cedex, France

2 CEA-DAM, DIF, F 91297, Arpajon, France

${ }^{3}$ Université de Valenciennes, LAMAV, LME. Mont Houy 59313 Valenciennes cedex 9, France

${ }^{4}$ LJK (Laboratoire Jean Kuntzmann) et LSP (Laboratoire de Spectrométrie Physique) Université Joseph Fourier - BP 53 38041 Grenoble Cedex 9, France
}

(C) EDP Sciences, SMAI 2009 


\section{INTRODUCTION}

The framework is the Lagrangian formalism on unstructured meshes where several fluids are present in the flow. We are interested in the Euler equations of gas dynamics (1.0.1) written below by way of the convective time derivative, the other differential operators are related to a fixed cartesian frame.

$$
\left\{\begin{array}{c}
\rho \frac{d \tau}{d t}-\vec{\nabla} \cdot \vec{u}=0 \\
\rho \frac{d \vec{u}}{d t}+\vec{\nabla} p=0 \\
\rho \frac{d E}{d t}+\nabla \cdot \overrightarrow{p u}=0
\end{array}\right.
$$

where $\tau=1 / \rho, \rho$ the density, $\vec{u}$ velocity and E the total energy of the flow and $\frac{d q}{d t}:=\partial_{t} q+\vec{u} \vec{\nabla} q$ for arbitrary scalar function.

We particulary want to study some numerical problems arising when these Euler equations of gas dynamics (1.0.1) are coupled to turbulence to produce dynamic mixing of fluids.

The overall problem is the introduction of a second order turbulent mixing model [14]. Because such model is quite complex and its complete formulation is not necessary for our present study, one defines an algebraic closure for quantities defining the dynamics of the turbulence $\left(R_{i j}, k, \varepsilon, \overline{u "}\right.$, etc...). It corresponds to a stressstrain relation that allows to couple the average flow quantities to a system of unsteady equations for mass concentrations and their turbulent fluxes. The underlying model is linear and depends on coefficients which are calculated in terms of the purely hydrodynamic splitted Lagrange-ALE step on two-dimensional unstructured meshes. This problem requires to consider a turbulent stress tensor and a diffusion term whose coefficient is proportional to this tensor which varies in time and space.

When the cells are too distorted, the Lagrangian formalism is no longer reliable with respect to accuracy, consistency and stability and the use of a rezoning process to adjust the grid (Arbitrary-Lagrangian-Eulerian) is necessary. This step allows to obtain a better grid (smoothness, width) in order to improve the numerical accuracy of the first order (hyperbolic) operator and second order (anisotropic diffusion) operator of the coupled system.

The mesh adaptation is based on two treatments. The r-adaptation (see [5]) of the moving grid will be done by scalar weights and/or tensors, depending on the physics (velocity, mass concentrations and/or pressures). The h-adaptation (see [5]) will also reflect the local nature of the flow, and a major point here is the determination of the best topological cells in a zone to be remeshed, for that we use the approach described in [7] that deals with arbitrary convex polygonal remeshing. We emphasize that each new extensive unknown (such as density) needs to be defined in such a way that the overall process preserves conservation. A major constraint in our approach is to use a positive scheme for the diffusion process to avoid "entropy violation" on arbitrary star shaped polygonal meshes (inside each cell, it exists at least a point $x^{*}$ such that any edge e can be joined to $x^{*}$ by a segment included in that cell).

The paper is organized as followed, in a first section, we explain a modeling that we want to take into account in our flow. After what we recall the diffusion scheme we have used for simplicial mesh [9] and we present our approach to take into account arbitrary star shaped polygonal mesh by cutting it in simplices while using the scheme described in [9]. Then by way of test problems we show that the numerical order of our procedure applied on static mesh exceeds one. We also gives results on Lagrange-ALE mesh concerning weak coupling between hydrodynamics and turbulence. Finally, in the last section we draw some perspectives of our algorithm. 


\section{A Reduce Modeling}

\subsection{Stress-strain relation}

Turbulent mixing occurs when fluids of different characteristics are embedded in a highly perturbed flow whose averaged quantities are described by turbulence modelling. Recent progress in such area leads to second order model for the Reynolds stress and hyperbolic heat type equation for mass concentrations. That physical mechanism allows a better description of the way fluids are distributed in space and provides necessary accurate informations to improve the equation of state of the mixing state and to compute reaction rates of species compounding each of the fluids. The resulting set of equations is known to be tremendously complex and deserve specific developpement beyond the aim of the present paper. We focus our attention to two distinct behaviors of the whole modeling:

(1) the hyperbolic part needs special care on a lagrangian grid because: the accuracy is hard to determine, the stability depends directly on the discretization of gradient operator especially when strong shocks occurs.

(2) the turbulent diffusion process is non uniformly anisotropic and is discretized on non cartesian meshes.

For sake of simplicity we choose to reduce the model to an artificial strain-stress relation that allows to express directly a Reynolds stress in proportion to the dynamics of the flow. That impacts momentum and energy:

$$
\left\{\begin{array}{c}
\rho \frac{d \vec{u}}{d t}+\vec{\nabla} p+\nabla \cdot \overline{\bar{R}}=0 \\
\rho \frac{d E}{d t}+\nabla \cdot \overrightarrow{p u}+\nabla \cdot \overline{\bar{R}} \vec{u}=0 .
\end{array}\right.
$$

and the governing equation of mass concentration $c_{\alpha}$ of a fluid $\alpha$ :

$$
\rho \frac{d c_{\alpha}}{d t}-\nabla \cdot\left(C_{c^{\prime \prime}} \rho \frac{k}{\eta} \overline{\bar{R}} \cdot \vec{\nabla} c_{\alpha}\right)
$$

More precisely, with:

$$
\left\{\begin{array}{l}
\overline{\bar{\varepsilon}}=\frac{1}{2}\left[\vec{\nabla} \vec{u}+(\vec{\nabla} \vec{u})^{T}\right] \\
\overline{\bar{d}}=\overline{\bar{\varepsilon}}-\frac{1}{3}(\nabla \cdot \vec{u}) \overline{\overline{I d}}
\end{array}\right.
$$

One defines the Reynolds stress as:

$$
\left\{\begin{array}{c}
\overline{\bar{R}}=\frac{2}{3} \rho k \overline{\bar{I}}+\rho \nu_{T} \overline{\bar{d}} \\
\nu_{T}=c_{s}^{2} L_{s}^{2} \sqrt{\overline{\bar{d}}: \overline{\bar{\varepsilon}}} \\
k=\frac{\nu_{T}^{2}}{c_{s} L_{s}}, \eta=\frac{k^{3 / 2}}{c_{s} L_{s}}
\end{array}\right.
$$

$c_{s}$ and $C_{c}$ " are given coefficients, $L_{s}$ a characteristic length of the computational domain. 


\subsection{Diffusion operator}

For each fluid $\alpha$ one defines mass concentration $c_{\alpha}$ as the ratio of mass $m_{\alpha}$ of fluid $\alpha$ and total mass of fluids contained in a control volume: $m=\sum_{\gamma} m_{\gamma}$. Usual first gradient closure for $c_{\alpha}$ reads:

$$
\left\{\begin{array}{l}
\rho \frac{d c_{\alpha}}{d t}+\nabla \cdot \overrightarrow{F_{\alpha}}=0 \\
\vec{F}_{\alpha}=-C_{c}, \rho \frac{k}{\eta} \overline{\bar{R}} \cdot \vec{\nabla} c_{\alpha}
\end{array}\right.
$$

The diffusion coefficient $C_{c "} \rho \frac{k}{\eta} \overline{\bar{R}}$ is a non-spherical tensor and can be written as $C_{c}{ }^{\prime \prime} \frac{\left(c_{s} L_{s}\right)^{2}}{\nu_{t}} \overline{\bar{R}}$ (see (2.2.1) above). Notice, that a simplified version could be to only take into account isotropic diffusion by way of $\operatorname{Trace}(\overline{\bar{R}})=\frac{2}{3} \rho k$.

- For the present paper, we will only focus on a weak coupling for which hydrodynamic quantities modifies the diffusion tensor but we do not consider that the solution of diffusion influence the hydrodynamics.

- We will not focus on the discretization of the hydrodynamic part and we refer the reader of [7] where we use the overall approach and re-use the same code [2].

\section{MATHEMATICAL FRAMEWORK FOR SCALAR DIFFUSION PROCESS}

This section is devoted to give a framework for the simplified linear anisotropic time dependent problem. For a computational domain $\Omega \in \mathbb{R}^{2}$ with $\partial \Omega=\partial \Omega_{\text {dir }} \cup \partial \Omega_{\text {neu }}$ (neu and dir stand respectively for "Neumann" and "Dirichlet"):

$$
\begin{cases}\frac{\partial c}{\partial t}-\nabla \cdot(\overline{\bar{D}}(t, x) \vec{\nabla} c)=0 & \text { in } Q:=(0, \mathbb{T}) \times \Omega \\ c(0, x)=c_{0}(x) & \text { in } \Omega \\ c(t, x)=c^{d i r}(t, x) & \text { on }(0, \mathbb{T}) \times \partial \Omega_{\text {dir }} \\ -\overline{\bar{D}}(t, x) \vec{\nabla} c(t, x) \cdot n=c^{\text {neu }}(t, x) & \text { on }(0, \mathbb{T}) \times \partial \Omega_{\text {neu }}\end{cases}
$$

$\overline{\bar{D}}$ is called the diffusion tensor, $\overline{\bar{D}}$ is symmetric and positive defined

$$
\overline{\bar{D}}(t, x)=\left(\begin{array}{ll}
\alpha(t, x) & \beta(t, x) \\
\beta(t, x) & \gamma(t, x)
\end{array}\right), \quad \text { with }\left\{\begin{array}{l}
\alpha(t, x)>0 \text { and } \\
\alpha(t, x) \gamma(t, x)>(\beta(t, x))^{2} .
\end{array}\right.
$$

An important property for the scalar solution in whole space or with the homogeneous Neumann condition $c^{\text {neu }}=0$ (or the energy $\left|c^{2}\right|$ associated to (3.0.2) with $c^{d i r}=0, c(t,.) \in H_{0}^{1}(\Omega)$ ) of physical interest for such conservative scalar equation is the monotonicity:

$$
u(t=0, x) \geq v(t=0, x) \Longrightarrow \forall t>0, \quad u(t, x) \geq v(t, x) .
$$

In whole space, a semi-group approach in $L^{1}$ see e.g. [3] for conservative translation-invariant equation $(\overline{\bar{D}}$ independent of $x$ ) (3.0.4) gives a maximum principle. For more general case (with boundary condition) see e.g. [6], the solution satisfies the following two conditions:

- Extremal condition see [10]: decay in time of spatial extrema and Maximum Principle (with boundary condition here):

$$
\min \left\{\left(c(s, .), c_{b}\right)\right\} \leq \min c(t, .) \leq c(t, .) \leq \max c(t, .) \leq \max \left\{\left(c(s, .), c_{b}\right)\right\} .
$$

where $t>s$ and $c_{b}$ are the boundary values of $\mathrm{c}$ over the interval $[\mathrm{s}, \mathrm{t}]$. 
- Positivity condition. This is a special case of (3.0.4) with $v(x) \equiv 0$ or special case of (3.0.5) with $c_{b} \geq 0$ since $c \equiv 0$ is obviously solution of (3.0.2) with natural boundary condition. Hence, positivity can be expressed as:

$$
u(0, x) \geq 0 \Longrightarrow \forall t>0, u(t, x) \geq 0 .
$$

For example, for a temperature or mass concentration evolving with respect to an heat equation, this constraint is required for a numerical scheme for arbitrary size/shape of the mesh. Positivity is our minimal requirement throughout this paper.

We can notice that there is an other approach to study continuous solution of fully nonlinear second order scalar PDE see e.g. [1]:

$$
\begin{cases}\frac{\partial u}{\partial t}+H\left(t, x, u, \nabla u, \nabla^{2} u\right)=0 & \text { in }(0, \mathbb{T}) \times \Omega \\ u(x, 0)=u_{0}(x) & \text { in } \Omega, \text { and some boundary condition on }(0, \mathbb{T}) \times \partial \Omega\end{cases}
$$

The main assumption on $\mathrm{H}$ is the so-called ellipticity:

$$
\forall(t, x, u, p) \quad H(t, x, u, p, M) \leq H(t, x, u, p, N) \quad u \in \mathbb{R}, p \in \mathbb{R}^{2}, M, N \in S^{s y m, 2 \times 2} \text { with } M \geq N,
$$

for which the definition of a solution (called viscosity-solution) is directly formulated under a maximum principle. A function $\mathrm{u}$ is called a viscosity solution of (3.0.7) if and only if it is:

- Sub-solution: $\forall \Phi(t, x) \in C^{2}(Q)$ if for all local maximum $\left(t^{*}, x^{*}\right)$ of $u-\Phi$ :

$$
\partial_{t} \Phi\left(t^{*}, x^{*}\right)+H\left(t^{*}, x^{*}, u\left(t^{*}, x^{*}\right), \nabla \Phi\left(t^{*}, x^{*}\right), \nabla^{2} \Phi\left(t^{*}, x^{*}\right)\right) \leq 0,
$$

- Super-solution: $\forall \Phi(t, x) \in C^{2}(Q)$ if for all local minimum $\left(t^{*}, x^{*}\right)$ of $u-\Phi$ :

$$
\partial_{t} \Phi\left(t^{*}, x^{*}\right)+H\left(t^{*}, x^{*}, u\left(t^{*}, x^{*}\right), \nabla \Phi\left(t^{*}, x^{*}\right), \nabla^{2} \Phi\left(t^{*}, x^{*}\right)\right) \geq 0 .
$$

Note that this definition is implicit with respect to extremal points, and that it is not obvious to take it into account directly inside a finite volume scheme. But we mention it for the study of parabolic equations more general than (3.0.2).

\section{PROPERTIES FOR DisCRETE NUMERICAL SCHEME}

In the spirit of [10] and to mimic (3.0.5), we want to impose the following property to the solution of (3.0.2) in case of implicit scheme with $n$ the time step number, $K$ the mesh element:

$$
\min \left\{\left\{c_{K^{\prime}}^{n+1}, K^{\prime} \in V(K)\right\}, c_{K}^{n}, c_{b}\right\} \leq c_{K}^{n+1} \leq \max \left\{\left\{c_{K^{\prime}}^{n+1}, K^{\prime} \in V(K)\right\}, c_{K}^{n}, c_{b}\right\}
$$

where $\mathrm{V}(\mathrm{K})$ is the neighborhood of cells sharing at least a node with cell $\mathrm{K}, c_{b}$ is the solution at boundary between time $\left[t^{n}, t^{n+1}\right]$.

Ideally, in our context we want a diffusion scheme that fulfils the following properties:

1 locally conservative.

2 consistent and convergent.

3 discrete maximum/minimum principle or the weaker positivity condition (3.0.6).

4 reliability on arbitrary convex polygonal cells ${ }^{1}$ with high aspect ratio and/or cells stretching.

5 compatible with heterogeneous and arbitrary anisotropic diffusion tensor (positive defined matrix).

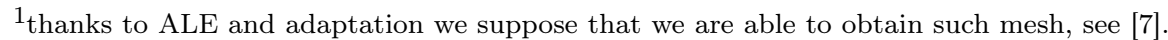


Many of these constraints have been taken into account in the paper [9] (see also [11] [15] for related results) but convergence has to be studied with respect to robustness. We recall their scheme in section 4.1.1 and explain our variant for polygonal cells in the next section 4.1.2.

\subsection{Positive solution for a diffusion problem with finite volume approximation}

\subsubsection{Positive solution of scheme [9] on simplicial mesh with arbitrary diffusion tensor}

We recall that the non-linear scheme of [9] corrects [11] in the case of general diffusion tensor. Integrating the equation (3.0.2) over a triangle $\mathrm{T}$ and using Green Formula, we obtain the evolution for the mean value:

$$
\partial_{t} \bar{c}_{T}+\frac{1}{|T|} \int_{\partial T} q . n d s=0, \quad q:=-\overline{\bar{D}} \nabla c
$$

and so the following balance:

$$
\partial_{t} \bar{c}_{T}+\frac{1}{|T|} \sum_{e \in \partial T} q_{e} \cdot n_{e}=0, \quad n_{e} \text { outward normal of e(T) of length }\left|n_{e}\right|=|e| .
$$

Using notation $c_{T}$ instead of $\bar{c}_{T}$, we use an implicit time stepping with time step $\Delta t$ :

$$
c_{T}^{n+1}+\frac{\Delta t}{|T|} \sum_{e \in \partial T} q_{e}^{n+1} \cdot n_{e}=c_{T}^{n}
$$

A two point flux approximation is considered: $q_{e}$ (we omit the time dependence knowing it is implicit), for each cell $\mathrm{T}$, only one degree of freedom is used and is noted $c_{T}$ (corresponding to $\bar{c}_{T}$ ). Hence, a two-point flux approximation uses only two degrees of freedom $c_{T^{+}}$and $c_{T^{-}}$(in short $c_{+}, c_{-}$) per each edge e (see Figure 1):

$$
q_{e} \cdot n_{e}=A_{e}^{+} c_{+}-A_{e}^{-} c_{-}
$$

where coefficients $A_{e}^{ \pm}$characterizes the scheme and depends on unknowns c itself as we will see. In the construction, there are mainly three steps:

- Step 1 - Definition of the unique collocation point $x_{T}$ in each simplex T.

Let $v_{1}, v_{2}, v_{3}$ be the vertices, the intersection of the three $\overline{\bar{D}}$-bisector lines defined the collocation point:

$$
x_{T}=\sum_{i=1}^{3} v_{i} \lambda_{i}, \quad \lambda_{i}=\frac{\left|e_{\theta_{i}}\right|_{\bar{D}}}{\sum_{j=1}^{3}\left|e_{j}\right|_{\bar{D}}}, \quad \text { where }|e|_{\bar{D}}:=\left|\overline{\bar{D}}^{1 / 2} e\right| .
$$

Here $e_{\theta_{i}}$ denotes the edge opposite to vertex $v_{i}$, hence $c_{T}$ represent now the value of $\mathrm{c}$ at $x_{T}$.

- Step 2 - Local non-linear diamond scheme (half edge flux $q_{e, i}, i=1,2$ computed by local Green formulas and non-linear interpolation):

For an interior edge e, $\mathrm{m}$ is defined as the midpoint. Let consider $T^{+}, T^{-}$the two triangles sharing e and $\overline{\bar{D}}^{+}$(resp. $\overline{\bar{D}}^{-}$) the constant metric tensor in $T^{+}$(resp. $\left.T^{-}\right)\left(\overline{\bar{D}}^{+}\right.$and $\overline{\bar{D}}^{-}$are not supposed to be equal). The two collocations points are denoted by $x^{+}, x^{-}$. The edge e and the midpoint $\mathrm{m}$ split the quadrilateral $v_{1} x^{+} v_{2} x^{-}$(the diamond cells) into four triangles $T_{i}^{ \pm}, i=1,2$ see Figure 1 . The local normal vector $n_{m}^{ \pm}$and $n_{e, i}, i=1,2$ are the normal to intervals $\left[m ; x_{ \pm}\right]$and $\left[m ; v_{i}\right], i=1,2$. Their length is assumed to be equal to the length of the underlying intervals, hence for example: $n_{m}^{+}=\left|x^{+}-m\right|$ and then $n_{e, i}=\frac{1}{2} n_{e}$. Hence, we get the following identity $n_{1}^{ \pm}+n_{m}^{ \pm}+\frac{1}{2} n_{e}=0$. Now applying a Green 


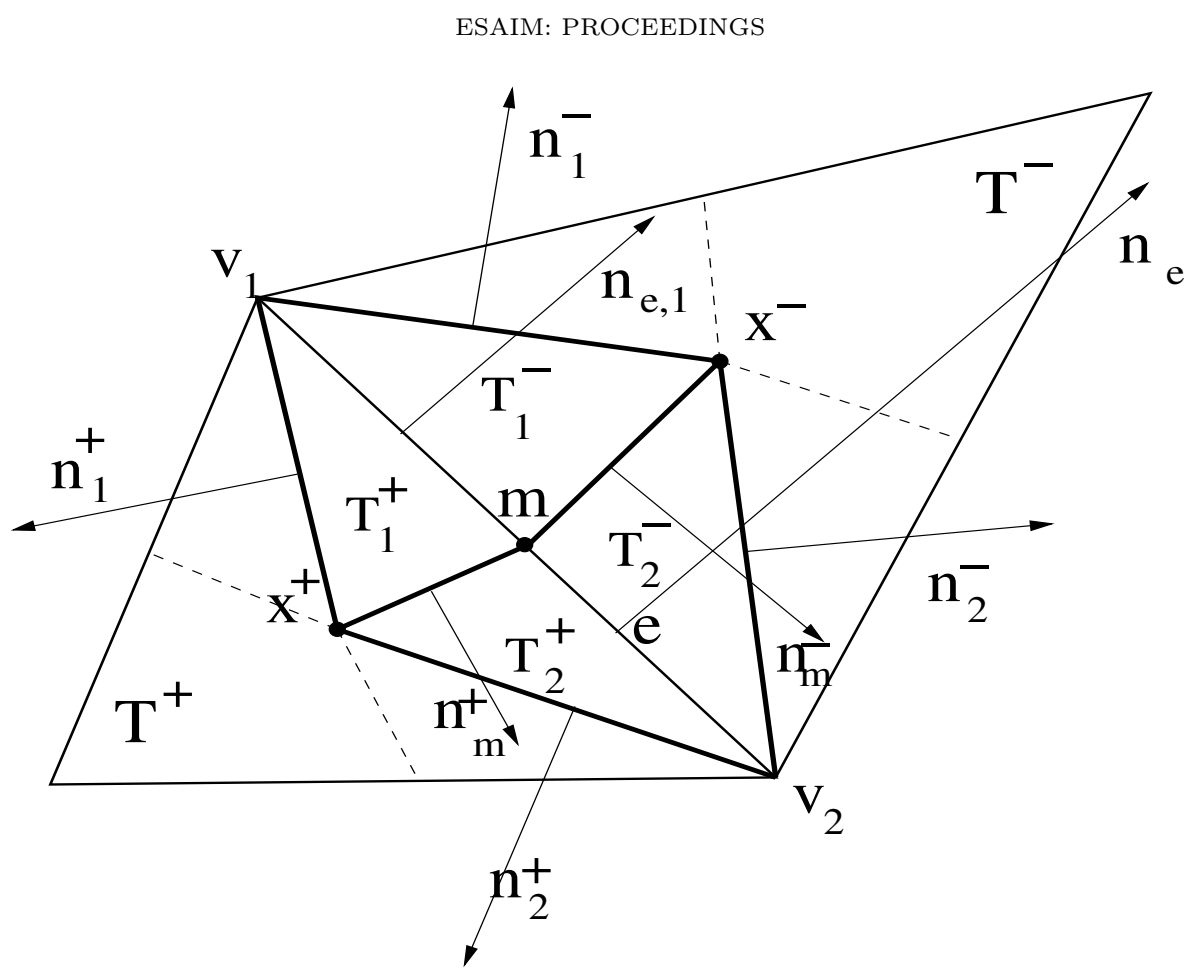

Figure 1. Notations for all local problems used for green-formula in sub-cells $T_{i}^{ \pm}, i=1,2$ and non-linear local underlying reconstruction of scheme [9]. The overall scheme will finally use both primal cell, diamond cell (around edge e) and dual cell around each vertex $v_{i}$.

formula in each of this triangles:

$$
\int_{T} \overline{\bar{D}}^{-1} q d x=-\int_{\partial T} c n d s
$$

for which a midpoint formula is applied for both terms, for example for the $T_{1}^{+}$:

$$
-\left|T_{1}^{+}\right|\left(\overline{\bar{D}}^{+}\right)^{-1} q_{e, 1}^{+}=\frac{c_{1}+c_{+}}{2} n_{1}^{+}+\frac{c_{1}+c_{m}}{2} n_{e, 1}+\frac{c_{+}+c_{m}}{2} n_{m}^{+}
$$

$c_{m}$ being the value at point $\mathrm{m}$. A similar formula holds for triangles $T_{1}^{-}$.

$$
-\left|T_{1}^{-}\right|\left(\overline{\bar{D}}^{-}\right)^{-1} q_{e, 1}^{-}=\frac{c_{1}+c_{-}}{2} n_{1}^{-}-\frac{c_{1}+c_{m}}{2} n_{e, 1}+\frac{c_{-}+c_{m}}{2} n_{m}^{-}
$$

Taking into account the continuity of normal flux across edge e:

$$
q_{e, 1}^{+} \cdot n_{e}=q_{e, 1}^{-} \cdot n_{e} \equiv q_{e, 1} \cdot n_{e}
$$

$c_{m}$ is eliminated from (4.1.7) and (4.1.8). The same is done for second half flux $q_{e, 2} . n_{e}$ and finally with the following notation: $k_{ \pm}^{i}=\overline{\bar{D}}^{ \pm} n_{i}^{ \pm} \cdot n_{e}, \mathrm{i}=1,2$ and $d_{ \pm}=\frac{1}{2} \bar{D}^{ \pm} n_{e} \cdot n_{e}$, then the two (still unknown) half 
flux writes:

$$
\left\{\begin{array}{l}
q_{e, 1} \cdot n_{e}=\frac{c_{+} d_{+} k_{-}^{1}+c_{-} d_{-} k_{+}^{1}-c_{1}\left(d_{+} k_{-}^{1}+d_{-} k_{+}^{1}\right)}{2\left(\left|T_{1}^{+}\right| k_{-}^{1}-\left|T_{1}^{-}\right| k_{+}^{1}\right)} \\
q_{e, 2} \cdot n_{e}=\frac{c_{+} d_{+} k_{-}^{2}+c_{-} d_{-} k_{+}^{2}-c_{2}\left(d_{+} k_{-}^{2}+d_{-} k_{+}^{2}\right)}{2\left(\left|T_{2}^{+}\right| k_{-}^{2}-\left|T_{2}^{-}\right| k_{+}^{2}\right)}
\end{array}\right.
$$

and a convex combination of this two half fluxes is considered:

$$
q_{e} \cdot n_{e}=\mu_{1} q_{e, 1} \cdot n_{e}+\mu_{2} q_{e, 2} \cdot n_{e}, \quad \mu_{1}+\mu_{2}=1 .
$$

If it verifies a two point flux approximation $q_{e} \cdot n_{e}=A_{e}^{+}(c) c_{+}-A_{e}^{-}(c) c_{-}$, so the resulting solution of (4.1.10) (4.1.11) for internal edges:

$$
\left\{\begin{array}{l}
A_{e}^{+}(c)=\mu_{1} \frac{d_{+} k_{-}^{1}}{2\left(\left|T_{1}^{+}\right| k_{-}^{1}-\left|T_{1}^{-}\right| k_{+}^{1}\right)}+\mu_{2} \frac{d_{+} k_{-}^{2}}{2\left(\left|T_{2}^{+}\right| k_{-}^{2}-\left|T_{2}^{-}\right| k_{+}^{2}\right)} \\
-A_{e}^{-}(c)=\mu_{1} \frac{d_{-} k_{+}^{1}}{2\left(\left|T_{1}^{+}\right| k_{-}^{1}-\left|T_{1}^{-}\right| k_{+}^{1}\right)}+\mu_{2} \frac{k_{+}^{2}}{2\left(\left|T_{2}^{+}\right| k_{-}^{2}-\left|T_{2}^{-}\right| k_{+}^{2}\right)}
\end{array}\right.
$$

where $\quad \mu_{1}=\frac{\gamma_{2}}{\gamma_{2}-\gamma_{1}}, \mu_{2}=\frac{-\gamma_{1}}{\gamma_{2}-\gamma_{1}}, \quad$ with $\quad \gamma_{i}=\frac{c_{i}\left(d_{+} k_{-}^{i}+d_{-} k_{+}^{i}\right)}{2\left(\left|T_{i}^{+}\right| k_{-}^{i}-\left|T_{i}^{-}\right| k_{+}^{i}\right)}$

For boundary edges:

- On $\partial \Omega_{d i r}$ is imposed Dirichlet boundary condition $c=c^{d i r}$, the flux writes $q_{e} \cdot n_{e}=A_{e}^{+}(c) c_{+}+A_{e}^{-}(c)$ where:

$$
\left\{\begin{array}{l}
A_{e}^{+}(c)=-\frac{1}{2\left|T^{+}\right|}\left(n_{1}^{+}+n_{2}^{+}\right) \cdot \overline{\bar{D}}^{+} n_{e} \\
A_{e}^{-}(c)=\frac{1}{2\left|T^{+}\right|}\left(c^{\operatorname{dir}}\left(v_{1}\right) n_{2}^{+}+c^{\operatorname{dir}}\left(v_{2}\right) n_{1}^{+}\right) \cdot \overline{\bar{D}}^{+} n_{e}
\end{array}\right.
$$

- On $\partial \Omega_{n e u}$, we impose Neumann boundary condition $-\overline{\bar{D}} \nabla c . n=c^{n e u}$, the flux writes:

$$
q_{e} \cdot n_{e}=\bar{c}_{e}^{n e u}\left|n_{e}\right|
$$

where $\bar{c}_{e}^{n e u}$ is a mean of $c^{n e u}$ on edge e.

- Step 3 - Interpolation of vertex values $c_{i}, i=1,2$ in non-linear flux (4.1.12). In [9], the authors tested the good behavior of the inverse distance weighting [13] of unknown values $c\left(x_{T}\right)$ for all $\mathrm{T}$ around each $v_{i}$, let $N\left(v_{i}\right)$ be this set. They claimed that interpolation is robust in case of non smooth solution but did not prove its second order accuracy.

$$
c_{i}=\sum_{T \in N\left(v_{i}\right)} c\left(x_{T}\right) w_{T}, \quad w_{T}=\frac{\left|x_{T}-v_{i}\right|^{-1}}{\sum_{T^{\prime} \in N\left(v_{i}\right)}\left|x_{T^{\prime}}-v_{i}\right|^{-1}}
$$

The non-linear system discretizing (4.1.3) with boundary condition (4.1.13) (4.1.14) is then $M(X) X=b$, where:

(1) the matrix $\mathrm{M}(\mathrm{X})$ of the discretization for the evolution equation writes:

$$
M(X):=I+\operatorname{Diag}\left(\frac{\Delta t}{|T|}\right) A(X), \quad \text { where } \quad \operatorname{Diag}\left(\frac{\Delta t}{|T|}\right)_{i j}=\frac{\Delta t}{\left|T_{i}\right|} \delta_{i j}
$$


and $\Delta t$ is the time step of Lagrangian step, $|T|$ surface of simplex $\mathrm{T}$, and $\mathrm{A}(\mathrm{X})$ the assembling matrix resulting of internal edges (4.1.12) and contributing part of Dirichlet boundary condition $A_{e}^{+}(c)$ in (4.1.13).

(2) The right hand side $b$ is such that each component (for each simplex):

$$
b_{T}=c_{T}^{n}-\frac{\Delta t}{|T|}\left(\sum_{e \in \partial \Omega_{d i r} \cap \partial T} A_{e}^{-}+\sum_{e \in \partial \Omega_{n e u} \cap \partial T} \bar{c}_{e}^{n e u}\left|n_{e}\right|\right) .
$$

The overall non-linear system $M(X) X=b$ is solved by Picard iteration:

$$
\left\{\begin{array}{l}
X^{0}=X^{*} \quad \text { such that } X_{T}^{*} \geq 0 \\
\text { Solve } M\left(X^{k-1}\right) X^{k}=b, \quad \text { until : } \\
\quad\left\|M\left(X^{k}\right) X^{k}-b\right\| \leq \epsilon_{n o n}\left\|M\left(X^{0}\right) X^{0}-b\right\|, \quad\left(\epsilon_{n o n}=10^{-10} \text { if nothing specified }\right)
\end{array}\right.
$$

Here, we choose to solve linear systems in (4.1.18) with GMRES algorithm with diagonal preconditioning. Iterations are terminated when relative norm w.r.t initial residual is lower that $10^{-14}$.

In [9], for stationary version of (3.0.2) (Poisson equation $-\nabla \cdot \overline{\bar{D}} \nabla u=f$ so that $\mathrm{M}(\mathrm{X})$ is replaced by $\mathrm{A}(\mathrm{X})$ in (4.1.16), and $c_{T}^{n}$ by $f_{K}$ in (4.1.17)), it is proved the following when (4.1.11)(4.1.12)(4.1.18) are used:

Properties 4.1. Let $b_{T} \geq 0, X_{T}^{*} \geq 0$, for all $T$, and linear systems in Picard iterations are solved exactly, then all iterates $X^{k}$ in (4.1.18) verifies:

$$
X_{T}^{k} \geq 0, \text { for all } T \text {. }
$$

Unfortunately the scheme does not verifies a Discrete Maximum Principle so that overshoot/undershoot or oscillations can appear in numerical solution (see [9] and in section 5.2).

\subsubsection{Positive solution on arbitrary convex polygonal mesh by cutting in triangles}

The overall scheme [9] does not behave so good if the mesh is composed of arbitrary polygonals, specially for stretched polygonal cells. The reason invoked is partially due to the fact that the construction of a collocation point ensuring the system to be positive is only limited to a restricted class of meshes and diffusion tensors. In our case, the constraint on diffusion tensor can be too strong (we do not control coefficients of the tensor) and our polygonal cells can be both stretched and may be constituted by more than four vertices (see numerical examples below). A study of how to adapt the mesh and obtain the collocation point such that it ensures positivity, is far from beeing an easy task. So that for the paper, we finally adopt the strategy that slices the arbitrary polygonal mesh into simplices. This is a sufficient operation that permits to deal with arbitrary anisotropic diffusion coefficient and any star shaped (and then convex) polygonal cells.

In brief, we do the following (see Figure 2):

- Let $\mathrm{M}^{\text {poly }}$ be an arbitrary convex polygonal mesh. For any cell $\mathrm{K}$ having more than three vertices we split it into simplices $T(K, i), i \leq \operatorname{card}(K), \operatorname{card}(K)$ the number of vertices in K. Each simplex is defined by two consecutive vertices linked to the gravity center of $\mathrm{K}$ (or a point for which the polygonal cell is star shaped), see Phase 1 in Figure 2 . We note $\mathrm{M}^{\mathrm{SMPL}(\text { poly) }}$ the resulting mesh constituted by only triangles. We transfer all quantities on the splitted triangle cells in a conservative way, at this moment, we only apply a first order transfer:

$$
c_{T(K, i)}^{n}=c_{K}^{n}, \quad \forall i=1, \ldots, \operatorname{card}(K) .
$$

- We solve the diffusion problem with the scheme presented in the previous subsection (4.1.11)(4.1.12)(4.1.18)

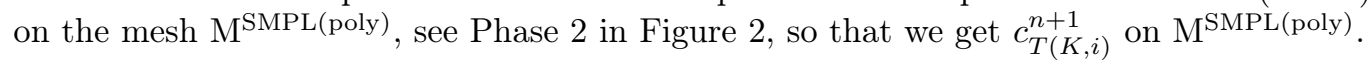




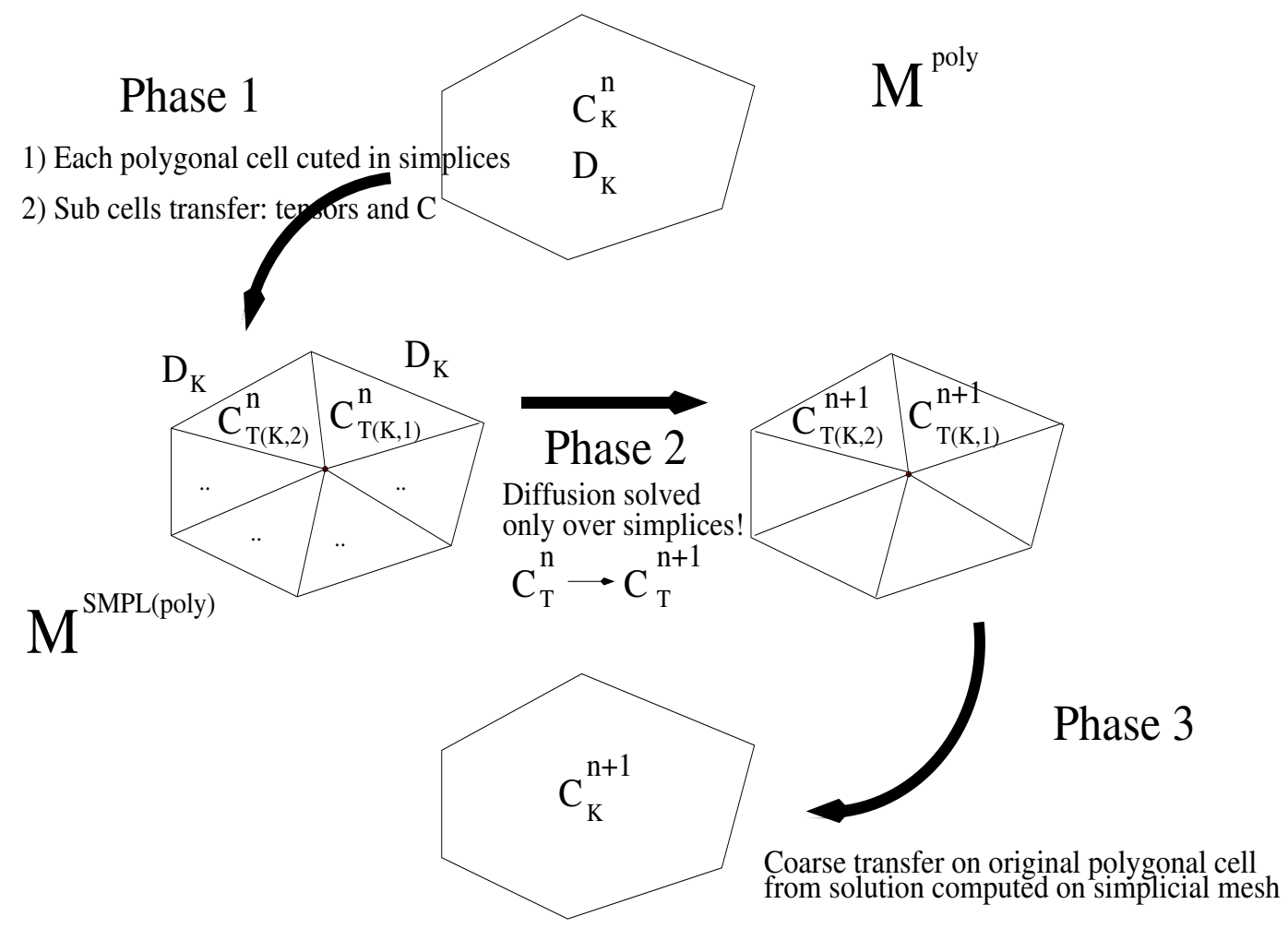

FiguRE 2. Strategy to obtain positive solution of (3.0.2) on arbitrary convex polygonal (or even better: star shaped polygonal) cells.

- We redefine the value on the initial polygonal cell on $M^{\text {poly }}$ by an area weighted averaging (conservative and first order):

$$
c_{K}^{n+1}=\frac{1}{|K|} \sum_{i}|T(K, i)| c_{T(K, i)}^{n+1},
$$

and we finally loop on global time problem.

Some comments on this approach:

- For the building of the slave simplicial mesh, we begin to add the barycenter of the polygon K. Next, inside each $\mathrm{K}$, we create a simplex by connecting two consecutive nodes of $\mathrm{K}$ with this new vertex. We do not know what sub-division should be better otherwise, and our strategy is to give an isotropic treatment, a drawback is that it makes the linear system much larger.

- Phase (4.1.20) of this approach must be improved, especially, it does not preserve linear field producing a loss of accuracy (see numerical section (5)), as a consequence it can not be of "second" order.

- When diffusion process is coupled to moving mesh problems, a way to obtain the mesh $\mathrm{M}^{\mathrm{SMPL}(\mathrm{poly})}$ at later time without redefining it "from scratch" is:

(1) to compute and save the connectivity of the first $\mathrm{M}^{\mathrm{SMPL}(\text { poly) }}$.

(2) to update the current position of vertices (connectivity of polygonal mesh $\mathrm{M}^{\text {poly }}$ does not change).

We have supposed that the unknown $\mathrm{c}$ is an extensive variable like density, but the result adapts easily to the Lagrangian framework (2.2.1) $\rho \frac{\partial c}{\partial t}$. 


\subsection{Positive solutions for a diffusion problem with linear finite element approximation}

Let $\Omega$ be a bounded open set of $\mathbb{R}^{2}, \mathbb{T}>0$ and $\overline{\bar{D}}$ a symmetric positive definite matrix. We use standard Sobolev spaces and notations. We want to find $u$ such that:

$$
\left\{\begin{array}{c}
\frac{\partial u}{\partial t}-\nabla \cdot \overline{\bar{D}} \nabla u=0 \\
u(x, 0)=U_{0}(x) \quad \text { in } \Omega, \quad \text { and }(0, \mathbb{T}) \times \Omega, \\
\text { some boundary condition on }(0, \mathbb{T}) \times \partial \Omega .
\end{array}\right.
$$

Let $\varphi \in H_{0}^{1}(\Omega)$ be a test function. Consider Neumann homogeneous boundary conditions. The classical weak formulation of the previous problem yields: consider $U_{0} \in L^{2}(\Omega)$ and find $u \in L^{2}(] 0, \mathbb{T}\left[; H_{0}^{1}(\Omega)\right) \cap C\left([0, \mathbb{T}] ; L^{2}(\Omega)\right)$ such that:

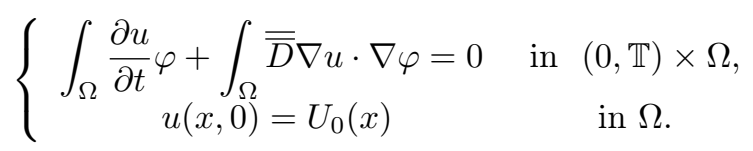

Consider a triangulation $\mathcal{T}_{h}, h>0$, of $\Omega$ and the standard discrete formulation of 4.2.1 using piecewise linear $\left(P_{1}\right)$ finite elements discretization. As it is known, the finite element approximation of diffusion problem does not always satisfy the maximum principle [4]. We are looking for sufficient conditions for positive solutions of problem (4.2.1) with respect to any given positive $U_{0}$. We use an implicit time step discretization. Let $\Delta t$ be the time step. Let $T$ be a triangle, $|T|$ its area and $v_{i}, v_{j}, v_{k}$ its vertices.

Proposition 4.1. For any positive $U_{0}$, the $P_{1}$ finite element approximation of the solution of (4.2.1) is positive if we have the following conditions:

$$
\left\{\begin{array}{c}
\left(\overline{\bar{D}}\left(\overrightarrow{v_{k} v_{i}}\right)^{\perp}\right) \cdot\left(\overrightarrow{v_{k} v_{j}}\right)^{\perp}>0 . \\
\Delta t \geq \frac{|T|^{2}}{6\left(\overline{\bar{D}}\left(\overrightarrow{v_{k} v_{i}}\right)^{\perp}\right) \cdot\left(\overrightarrow{v_{k} v_{j}}\right)^{\perp}} .
\end{array}\right.
$$

Where $\left(\overrightarrow{v_{k} v_{j}}\right)^{\perp}$ is the external normal to vector $\overrightarrow{v_{k} v_{j}}$ on $T$.

Proof. Let $\phi_{i}$ the nodal bases function associated with $v_{i}$ i.e., the continuous piecewise linear finite element function which equals 1 at the vertex $v_{i}$ and which vanishes at all other vertices of $\mathcal{T}_{h}$. Discrete formulation of (4.2.1) yields:

$$
\sum_{T \in \mathcal{T}_{h}} \int_{T}\left(\sum_{i=1}^{N} u_{i}^{n+1} \phi_{i} \phi_{j}+\Delta t\left(\overline{\bar{D}} \sum_{i=1}^{N} u_{i}^{n+1} \nabla \phi_{i}\right) \cdot \nabla \phi_{j}\right)=\sum_{T \in \mathcal{T}_{h}} \int_{T} \sum_{i=1}^{N} u_{i}^{n} \phi_{i} \phi_{j}, \quad j=1, \ldots, N
$$

Where $N$ is the number of degrees of freedom of $\mathcal{T}_{h}$.

Let $A x=b$ be the linear system obtained from (4.2.3). We want positive solutions of that system. The righthand side of (4.2.3) is always positive when $u_{i}^{n}$ is positive, so the vector $b$ has non-negative components. A very useful characterization of the matrix $A$ in this case is an M-matrix. In [12] page 30, we have a characterization of an M-matrix $A$ :

- Every diagonal entry of $A$ is positive: true in our case.

- Every off-diagonal entry of $A$ is non-positive:

if we look at this statement per triangle, then it is sufficient that

$$
\int_{T} \phi_{i} \phi_{j}+\Delta t\left(\overline{\bar{D}} \nabla \phi_{i}\right) \cdot \nabla \phi_{j} \leq 0
$$


To satisfy (4.2.4) as $\Delta t>0$, the calculus of $\nabla \phi_{l, l=i, j}$. and integration on $T$ gives the following sufficient condition:

$$
\left\{\begin{array}{l}
\Delta t \geq \frac{|T|^{2}}{6\left(\overline{\bar{D}}\left(\overrightarrow{v_{k} v_{i}}\right)^{\perp}\right) \cdot\left(\overrightarrow{v_{k} v_{j}}\right)^{\perp}}, \\
\left(\overline{\bar{D}}\left(\overrightarrow{v_{k} v_{i}}\right)^{\perp}\right) \cdot\left(\overrightarrow{v_{k} v_{j}}\right)^{\perp}>0 .
\end{array}\right.
$$

When $\overline{\bar{D}}$ is the identity matrix we have classical geometrical conditions on the three angles of $T$, see [4]: for each angle $\theta$ of $T, \theta \leq \frac{\pi}{2}+\epsilon, \epsilon>0$.

- $A$ have positive row sums:

if we look once more at this last statement per triangle, we obtain:

$$
\int_{T} \sum_{j}\left(\phi_{i} \phi_{j}+\Delta t\left(\overline{\bar{D}} \nabla \phi_{i}\right) \cdot \nabla \phi_{j}\right)>0
$$

The sum of the gradients of the nodal basis functions is always null on a triangle then,

$$
\int_{T} \sum_{j} \nabla \phi_{j}=0
$$

So, the previous inequality gives

$$
\int_{T} \phi_{i}^{2}>-\int_{T} \sum_{j \neq i}\left(\phi_{i} \phi_{j}\right)
$$

which is always true. 


\section{TeStS On PURELy DifFusion PROCESS ON SPLiTTED STATIC MESH}

Here, we test the scheme (4.1.11) (4.1.12) (4.1.18) used with the strategy (4.1.20) (4.1.21) for two test cases, the first one on cartesian grid and the second one on a Kershaw like mesh (see [8]).

\subsection{Accuracy tests}

We investigate the following problem:

$$
\left\{\begin{array}{lr}
\partial_{t} c=\Delta c & \text { in } \quad \Omega=(0,1) \times(0,1), \\
c=1 & \text { on } \partial \Omega,
\end{array}\right.
$$

with the initial condition: $c_{0}(x)=\sin (\pi x) \sin (\pi y)+1$. The exact solution reads:

$$
c^{e x}(t, x)=e^{-2 \pi^{2} t} \sin (\pi x) \sin (\pi y)+1 .
$$

In the two following test cases, we always take $n x=n y$ for the original cartesian grid (after which we can move the node to create non uniform quadrilaterals) and we note $h=\left(\max _{K}|K|\right)^{1 / 2}$ the characteristic mesh spacing (valid for any polygonal mesh), the time step being equals to $\Delta t=h^{2}$. We compute the error by the formula on the original non splitted mesh $e\left(t^{n}\right)=\sum_{K}|K|\left|c e^{e x}\left(t^{n}, x_{K}^{*}\right)-c_{K}^{n}\right|$, where $x_{K}^{*}$ is the gravity center of cell $\mathrm{K}$ and $c_{K}^{n}$ is the solution on the initial polygonal mesh, and we stop at final time $\mathbb{T}=0.1$.

On cartesian meshes (see Figure 3), the order of accuracy of the scheme is close to one and the computed solution maxima and minima tends toward continuous ones.
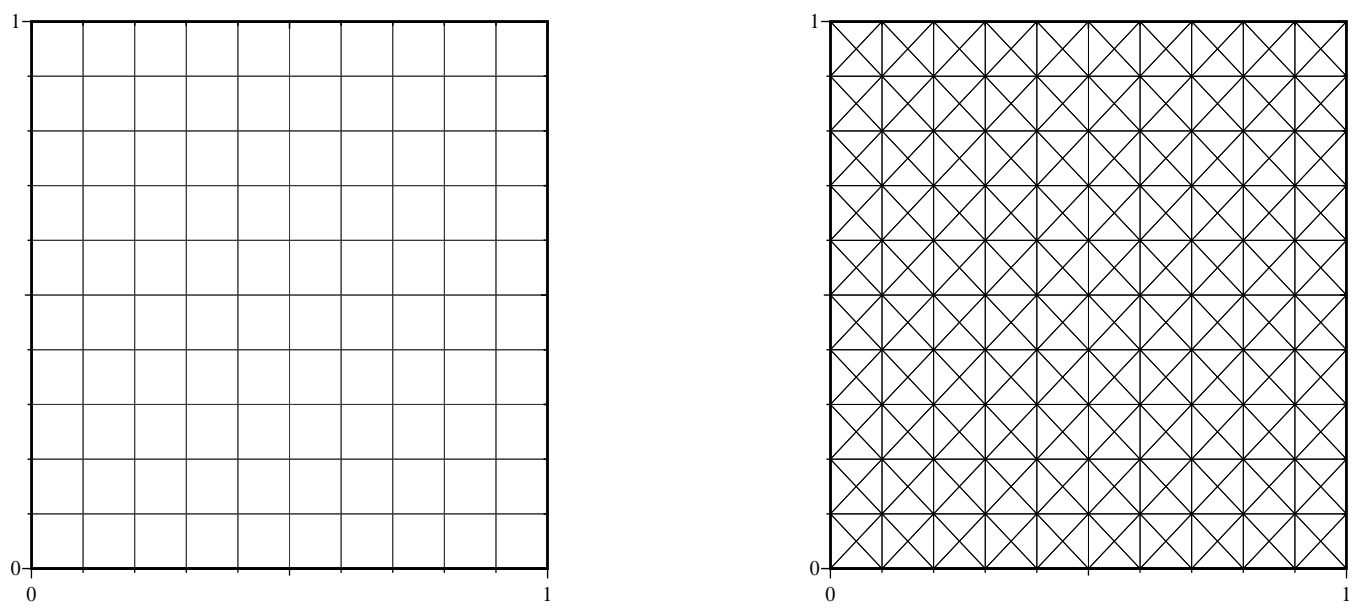

Figure 3. Cartesian grid ( with $\mathrm{nx}=\mathrm{ny}=11$ ) splitted in triangles

\begin{tabular}{c|c|c|c|c|c}
$\mathrm{nx}$ & $\mathrm{h}$ & error $\left(e_{h}\right)$ & numerical order $\left(\log _{2}\left(e_{h} / e_{h / 2}\right)\right)$ & exact $\min / \max$ & computed $\min / \max$ \\
\hline 11 & 0.1 & 0.024932 & - & $1.0034 / 1.13551$ & $1.00902 / 1.1786$ \\
21 & 0.05 & 0.00977574 & 1.35 & $1.00086 / 1.13806$ & $1.00204 / 1.15447$ \\
41 & 0.025 & 0.00418479 & 1.22 & $1.00021 / 1.1387$ & $1.00049 / 1.14541$ \\
81 & 0.0125 & 0.00191619 & 1.12 & $1.00005 / 1.13886$ & $1.00012 / 1.14182$
\end{tabular}


On Kershaw like mesh (stretched quadrilaterals: Wmesh, (see Figure 4)), the computed order of accuracy of the scheme is close to one, but surprisingly it is larger on the stretched mesh than on the cartesian mesh studied in Figure 3.
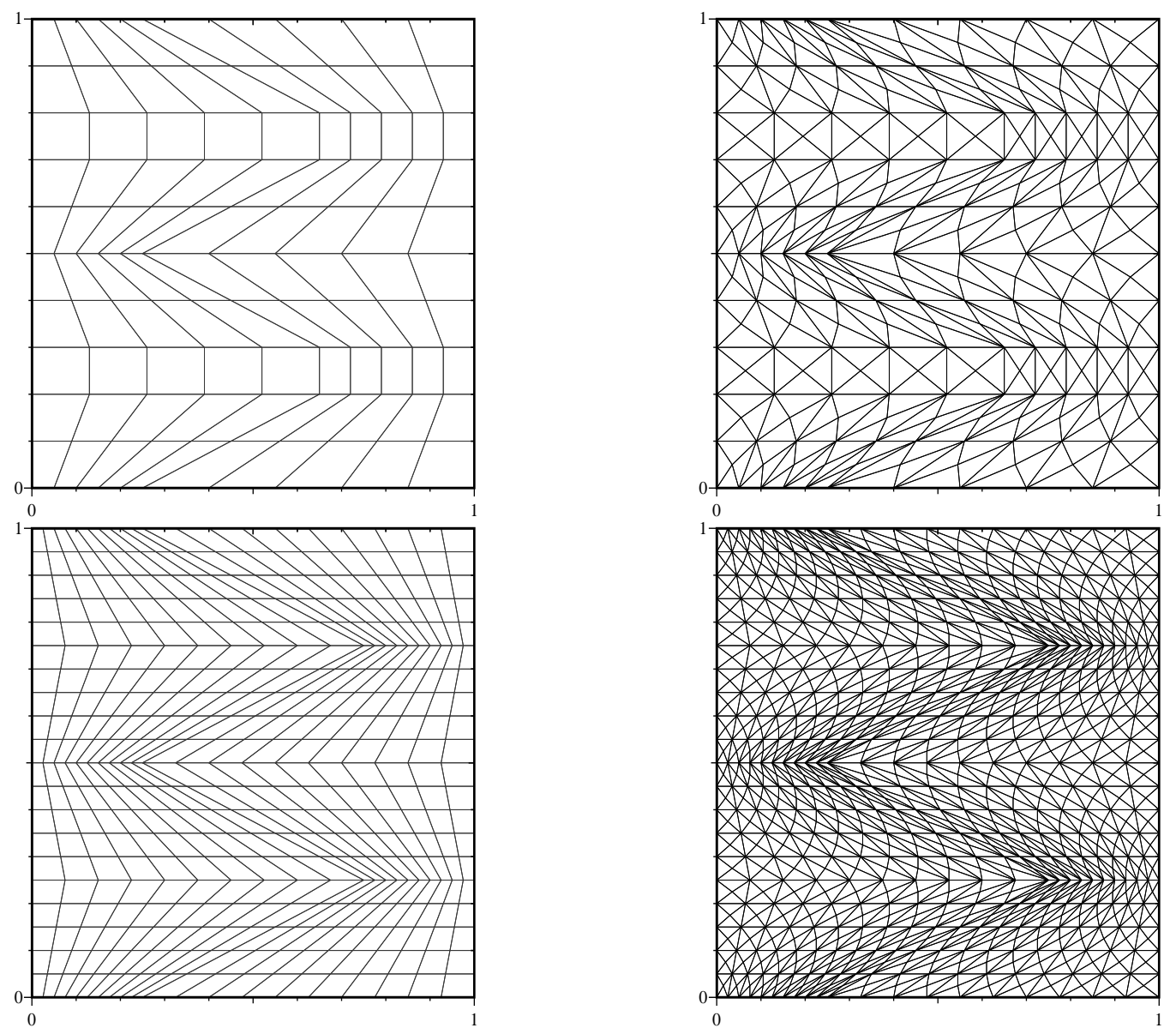

Figure 4. Kershaw like mesh (Left) and his resulting splitted triangular mesh (Right), Top Left with $\mathrm{nx}=\mathrm{ny}=11$, Bottom Left with $\mathrm{nx}=\mathrm{ny}=21$.

\begin{tabular}{c|c|c|c|c|c}
$\mathrm{nx}$ & $\mathrm{h}$ & error $\left(e_{h}\right)$ & numerical order $\left(\log _{h / h^{\prime}}\left(e_{h} / e_{h^{\prime}}\right)\right)$ & exact $\min / \max$ & computed $\min / \max$ \\
\hline 11 & 0.114018 & 0.0286646 & - & $1.00238 / 1.13583$ & $1.007 / 1.18885$ \\
21 & 0.0591608 & 0.0109641 & 1.4647778 & $1.00051 / 1.13806$ & $1.00147 / 1.15745$ \\
41 & 0.030104 & 0.00382034 & 1.5605168 & $1.00012 / 1.13873$ & $1.00032 / 1.14424$ \\
81 & 0.0151812 & 0.00136905 & 1.4990097 & $1.00003 / 1.13885$ & $1.00007 / 1.14$
\end{tabular}

We found out weird results: the rate of convergence of the scheme is low and a poor grid quality improves the accuracy. We recall that in paper [9] no convergence result is shown on cartesian grid for the interpolation function (4.1.15) with regular solution even with isotropic diffusion coefficient. In [9] the only convergence data are given for regular solution with a linear interpolation over an "imaginary no-degenerate triangle" and in that case second order is obtained associated to a lack of robustness. This last fact motivates our choice of function (4.1.15) for our preliminary study on more complex flows (see section 6).

As a consequence more work is needed to improve the interpolation phase (cf. Step 3 of section 4.1.1). 


\subsection{Test on discrete maximum/minimum principle}

Now we want to study if the numerical scheme obeys the discrete maximum/minimum principle previously introduce in (4.0.11). We deal with the following problem:

$$
\begin{cases}\partial_{t} c=\Delta c & \text { in } \Omega=(0,1) \times(0,1), \\ c_{0}(x)=1-\chi_{\left\{(x-0.5)^{2}+(y-0.5)^{2}<0.1\right\}} & \text { in } \Omega \\ c=1 & \text { on } \partial \Omega .\end{cases}
$$

The value of $c_{0}$ is hence 0 inside a circle and 1 outside. On smooth test cases, the discrete maximum $/ \mathrm{minimum}$ principle (4.0.11) is always satisfied. Rather than a sinusoidal initial condition we chose here to study the diffusion of an initial discontinuity. We plotted on Figure 5 the initial condition discretized on the quadrilateral meshes and the triangles of the splitted meshes where the discrete maximum/minimum principle is not verified after one time step. The maximum/minimum discrete principle (4.0.11) is tested on the splitted mesh used for the diffusion scheme, rather than on the initial non-splitted mesh, in order to emphasize the effect of the diffusion scheme itself rather than the effects of the transfer of quantities from one mesh to the other. On Figure 5, we can see violations of the discrete maximum/minimum principle occurring in zones of steep gradient, around the perimeter of the circus. With the finer mesh $(\mathrm{nx}=\mathrm{ny}=41)$, we also notice that more triangles are affected by slight oscillations (see Figure 5 Bottom Right) in zones where the mesh is most distorted. We conclude from the latter observations that the discrete maximum/minimum principle can be violated by the diffusion scheme in zones of steep gradient (remember that with the sinusoidal initial condition this principle is satisfied), and more likely in zones where the mesh is highly distorted.


Figure 5. Kershaw like mesh with initial condition (Left) and his resulting splitted triangular mesh, triangles with discrete maximum/minimum principle (4.0.11) not respected in red (Right), Top with $n x=n y=21$, Bottom with $n x=n y=41$. 


\section{Tests on Coupling with Hydrodynamic in AlE and Adaptation context}

\subsection{Passive diffusion of a scalar on moving mesh}

We consider the domain $\Omega=(0,7) \times(0,3)$, where $n x=141, n y=61$. The initial condition for hydrodynamic is a perfect gas with $\gamma=5 / 3$ at rest and:

$$
\left\{\begin{array}{l}
\rho=1, P=0.25, \quad x<1, \quad \text { else } \\
\rho=1, P=0.1, \quad y<1.5, \text { else } \\
\rho=0.1, P=0.1 .
\end{array}\right.
$$

Symmetry boundary condition is imposed all over the boundary.
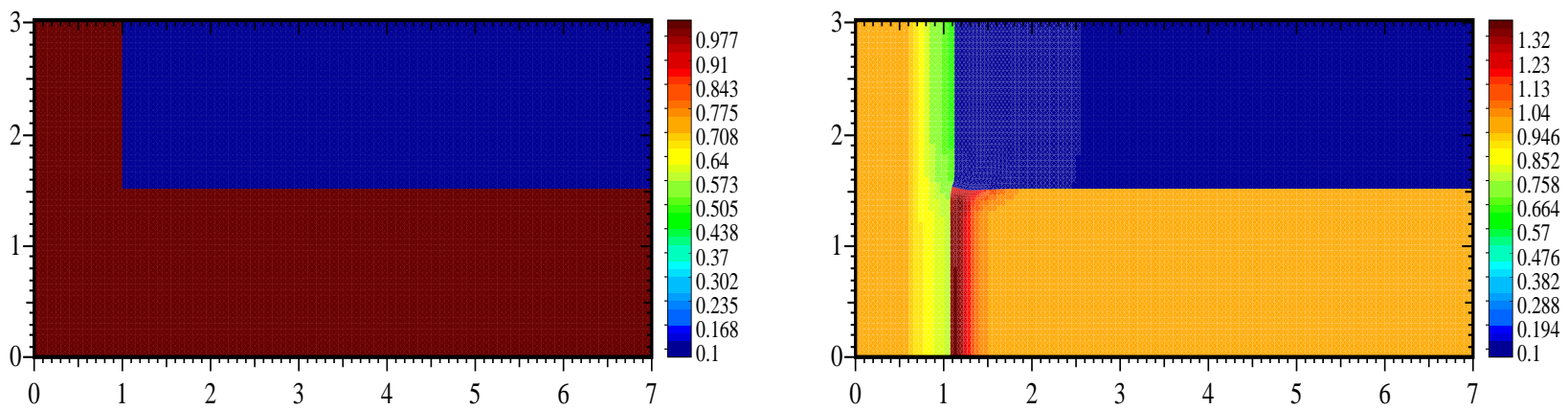

FiguRE 6. Initial density and density at time 0.539

We add a passive variable $\mathrm{c}(\mathrm{t}, \mathrm{x})$ that is diffused by way of (3.0.2), with $\overline{\bar{D}}=\overline{\bar{I}}$. The hydrodynamics process moves the polygonal mesh, so that the same diffusion process (isotropic heat equation) is solved on a different mesh at each time step. We take the following initial condition:

$$
c_{0}(x)= \begin{cases}1, & \text { on the square }[1.2,1.4] \times[1.35,1.55] \\ 0, & \text { else. }\end{cases}
$$

We choose homogeneous Neumann boundary condition all over the border. Figure 6 and 7 shows the flows behavior by way of density when the first hydrodynamic adaption phase has triggered (see [7]). At time 0.4, some quadrilaterals cells are modified to become polygonal with five or more nodes, after some time steps, this new adapted mesh on top/left in Figure 7 corresponds to the density in Figure 6 at time 0.539. The corresponding sliced triangular mesh used for the diffusion part is depicted on top/right in Figure 7.

We found out (see Figure 7 and 8) that the solution $c_{K}^{n}$ is truly positive all over the domain (at least at the maximum of residual for which linear systems are solved in Picard iteration). This is not affected with other kind of boundary conditions see Figure 9 . 


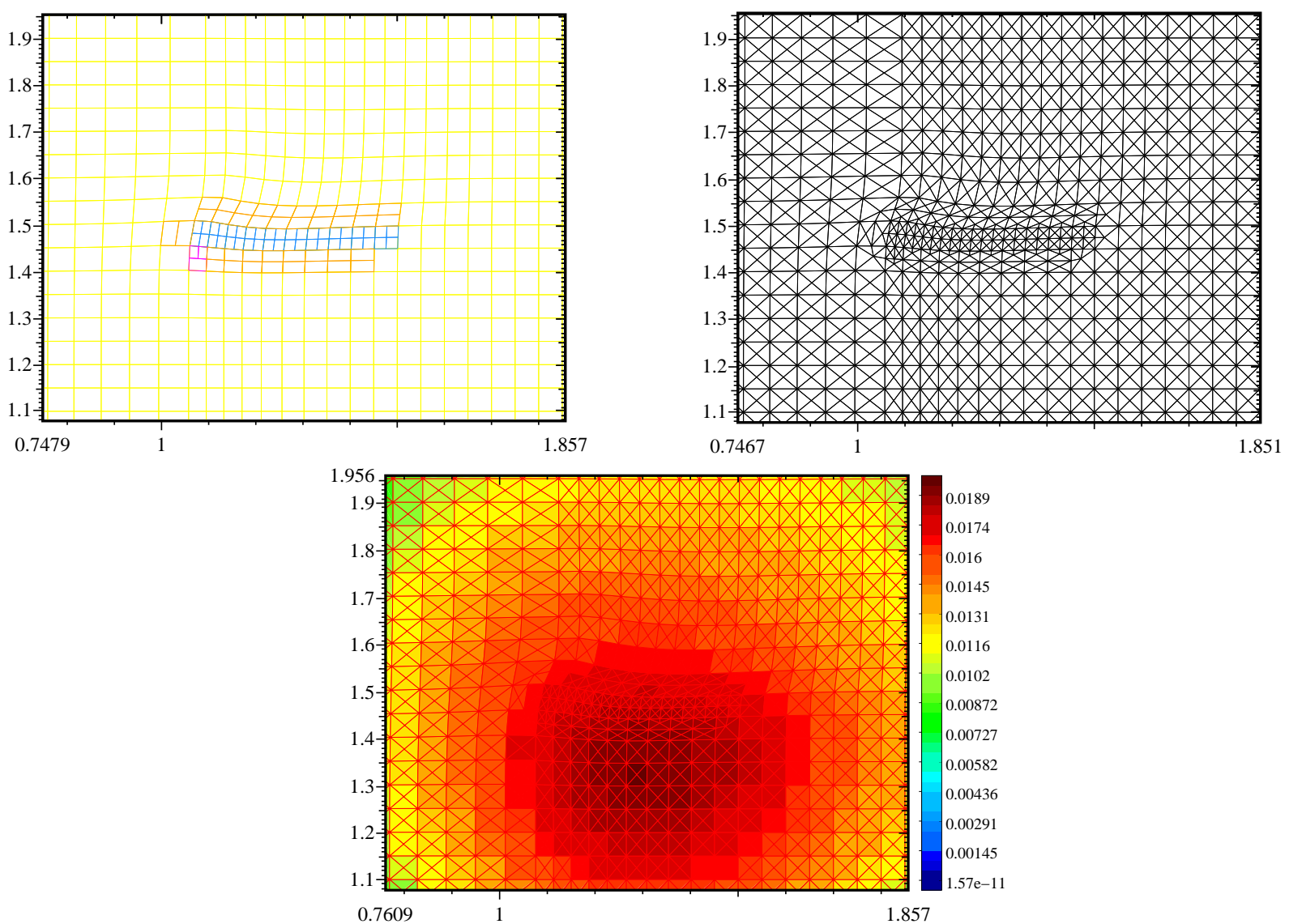

Figure 7. Time 0.539. A zoom on adapted polygonal cells (locally re-meshed of the initial moving cartesian grid) on top/left and the resulting splitted triangular cells on top/right), and numerical solution (bottom) of passively diffused quantity.
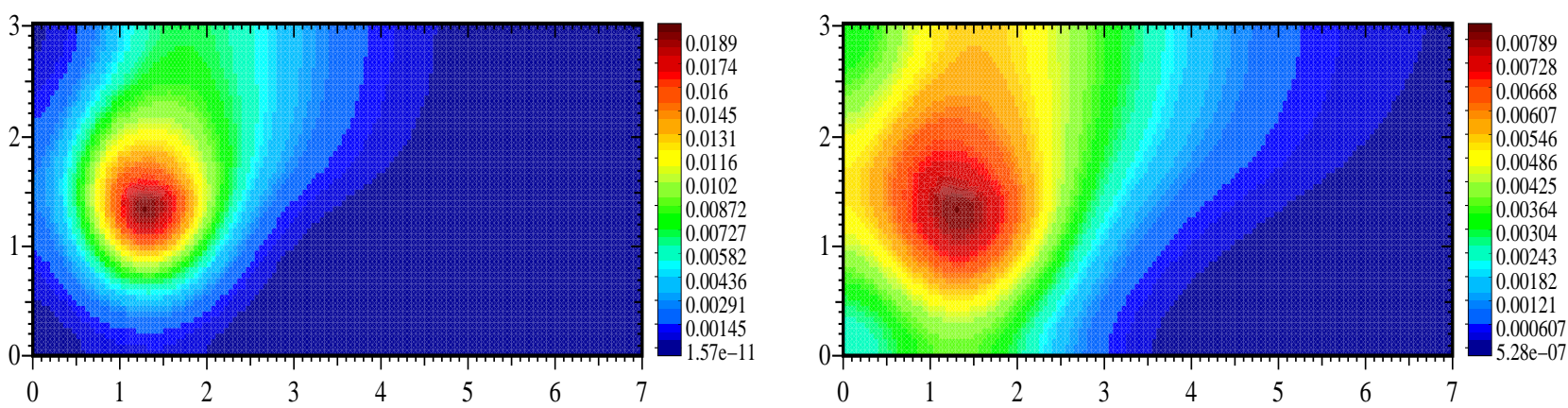

Figure 8. Positive solution of diffusion problem on entire domain at $\mathrm{t}=0.539$ (left), and $\mathrm{t}=0.79$ (right) (with homogeneous Neumann boundary condition). 

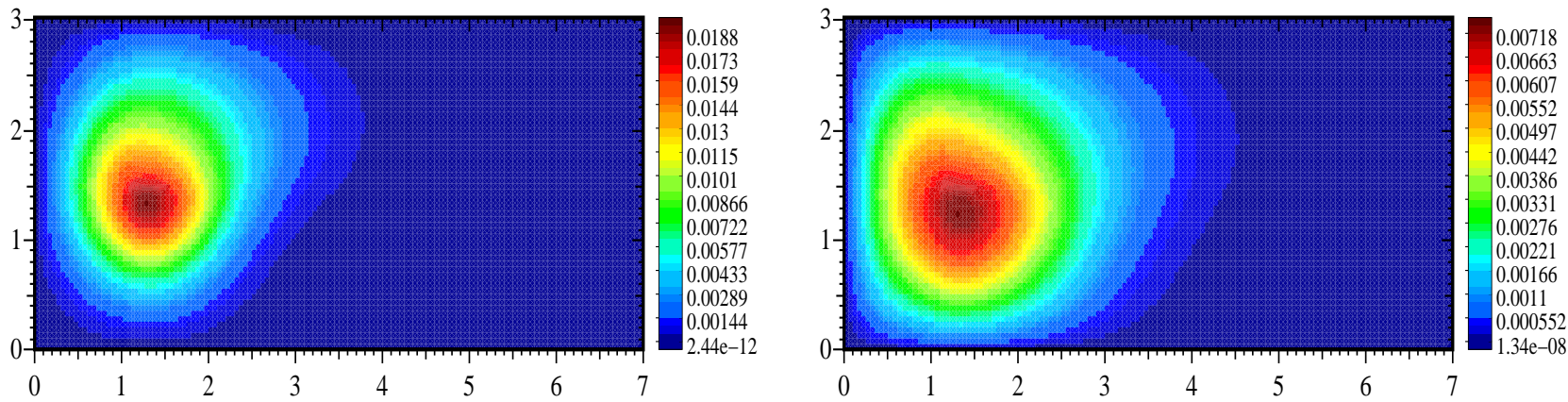

Figure 9. Positive solution of diffusion problem on entire domain at $\mathrm{t}=0.539$ (left), and $\mathrm{t}=0.79$ (right) with homogeneous Dirichlet condition at $\{\mathrm{y}=0\},\{\mathrm{y}=3\}$ and $\{\mathrm{x}=0\}$ and with homogeneous Neumann boundary condition on $\{\mathrm{x}=7\}$.

\subsection{Passive diffusion of a scalar on moving mesh with turbulent diffusion tensor}

In this test, we take the same problem of section 6.1 except that we take the tensor $\overline{\bar{D}}$ constructed from hydrodynamic quantities in (2.1.4)

$$
\overline{\bar{R}}=\frac{2}{3} \rho k \overline{\bar{I}}+\rho \nu_{T} \overline{\bar{d}}
$$

for which we add diagonal terms to ensure that the tensor is positive defined. We take the initial condition (6.1.2). For boundary condition, homogeneous Dirichlet boundary condition on top, left and bottom, and homogeneous Neumann condition at right border are imposed. For the Picard iteration (4.1.18), we take $\epsilon_{\text {non }}=10^{-13}$. Figure 10 gives the flow behavior in terms of velocity field. It shows zones where sharp gradients and shear are located producing Reynolds stress term.

We can check on Figure 11 that:

- the numerical solution of diffusion is positive.

- the diffusion is essentially limited to a region where hydrodynamic velocity is not uniform (see Figure $10)$. 

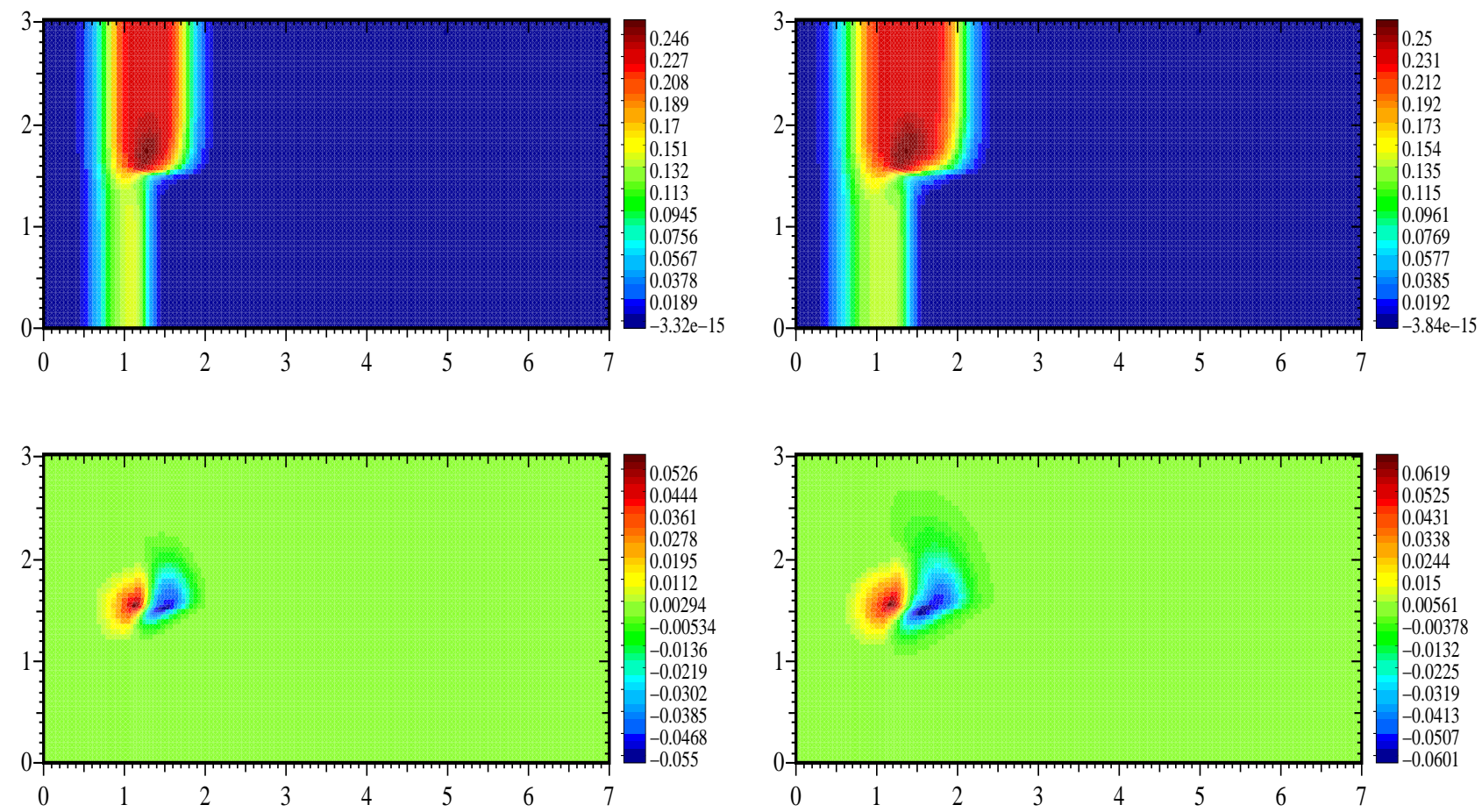

FIGURE 10. Numerical solution of hydrodynamic part: $\mathrm{x}$ (resp. y) component of velocity field at time $\mathrm{t}=0.539$ (left) and $\mathrm{t}=0.73$ (right) on top (resp. bottom).
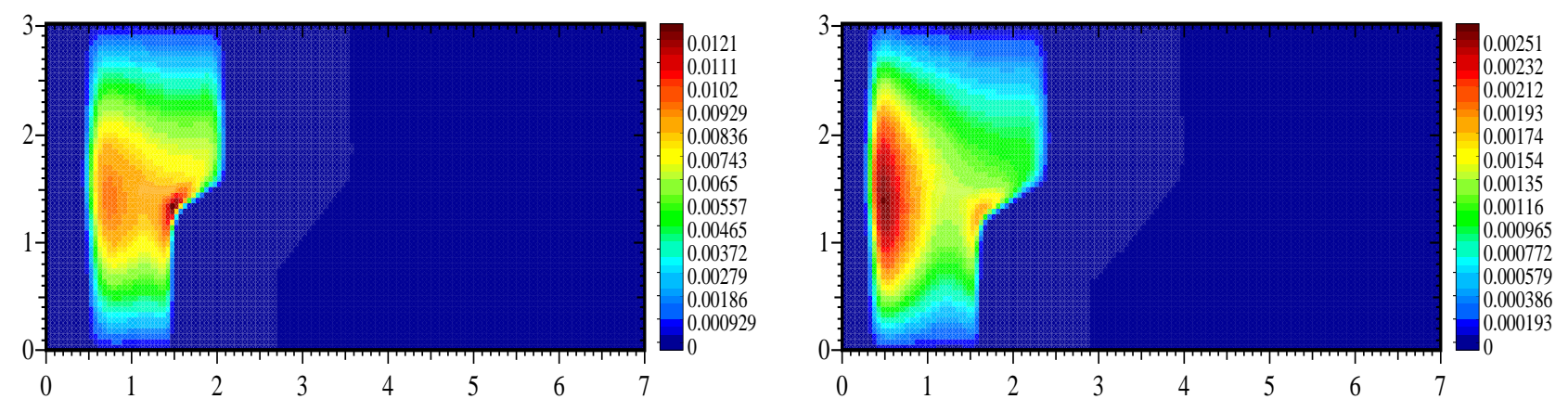

FIGURE 11. Numerical solution of diffusion process for weak coupling Hydrodynamic and Diffusion at time 0.539 (left) and $\mathrm{t}=0.73$ (right), here the diffusion tensor is computed from a stress relation (6.2.1). 


\section{Conclusion and extensions}

In this paper, we present a way to treat a weak coupling between hydrodynamics and diffusion problem on arbitrary polygonal mesh. The diffusion component part of the solution is computed on the moving mesh given by the hydrodynamics and the diffusion tensor is computed by a relation depending on the gradient of velocity of the flows to modelize a turbulent behavior.

Future works will include a way to obtain a diffusion scheme satisfying a good convergence behavior (see numerical tests in section 5) and then the Discrete Maximum Principle on arbitrary unstructured triangular mesh. With our alternative based on the partitioning of polygons in triangles, we could deal with star shaped polygonal cells (see also for instance the recent paper [15]). We will then come back to a strong coupling between diffused concentration and hydrodynamics.

\section{REFERENCES}

[1] G. Barles. Solutions de viscosité des équations de Hamilton-Jacobi. Springer, 1995.

[2] J.D. Benamou and P. Hoch. Go++ : A modular lagrangian/eulerian software for hamilton jacobi equations of geometric optics type. M2AN, 36(5):883-905, 2002.

[3] A. Chambolle and B.J. Lucier. A maximum principle for order-preserving mappings. C.R.A.S, 326:823-827, 1998.

[4] P.G. Ciarlet and P.A. Raviart. Maximum principle and uniform convergence for the finite element method. Comput. Methods Appl. Mech. Engrg., 2:17-31, 1973.

[5] P. Frey and P.L. George. Mesh Generation Application to Finite Elements. Hermes Science Publications, 2000.

[6] A. Friedman. Partial Differential Equations of the Parabolic Type. Prentice-Hall, 1964.

[7] P. Hoch, S. Marchal, Y. Vasilenko, and A.A. Feiz. Non conformal adaptation and mesh smoothing for compressible lagrangian fluid dynamics. ESAIM, 24:111-129, 2008.

[8] D. Kershaw. Differencing of the diffusion equation in lagrangian hydrodynamic codes. Journal Of Comput. Physics, (39):375395, 1981.

[9] K. Lipnikov, S. Shashkov, D. Svyatskiy, and Yu. Vassilevski. Monotone finite volume schemes for diffusion equations on unstructured triangular and shape-regular polygonal meshes. Journal Of Comput. Physics, (227):492-512, 2007.

[10] G.J. Pert. Physical constraints in numerical calculations of diffusion. Journal Of Comput. Physics, (42):20-52, 1981.

[11] C. Le Potier. Schéma volumes finis monotone pour des opérateurs de diffusion fortement anisotropes sur des maillages de triangles non-structurés. C.R. Acad. Sci., (Ser I 341):787-792, 2005.

[12] Alfio Quarteroni, Riccardo Sacco, and Fausto Saleri. Méthodes Numériques: Algorithmes, Analyse Et Applications. Springer Verlag, 2007.

[13] D. Shepard. A two-dimensional interpolation function for irregularly spaced data. In 23d ACM National Conference, pages 517-524, NY, 1968.

[14] O. Soulard and D. Souffland. A second order turbulent closure for modeling counter-gradient transport in variable density turbulent flows. ICHMT digital library, (1):369-372, 2006.

[15] Guangwei Yuan and Zhiqiang Sheng. Monotone finite volume schemes for diffusion equations on polygonal meshes. J. Comput. Phys., 227(12):6288-6312, 2008. 